\title{
The Perceived Tightness Scale Does Not Provide Reliable Estimates of Blood Flow Restriction Pressure
}

\author{
Zachary W. Bell, Scott J. Dankel, Robert W. Spitz, Raksha N. Chatakondi, \\ Takashi Abe, and Jeremy P. Loenneke
}

\begin{abstract}
Context: The perceived tightness scale is suggested to be an effective method for setting subocclusive pressures with practical blood flow restriction. However, the reliability of this scale is unknown and is important as the reliability will ultimately dictate the usefulness of this method. Objective: To determine the reliability of the perceived tightness scale and investigate if the reliability differs by sex. Design: Within-participant, repeated-measures. Setting: University laboratory. Participants: Twentyfour participants (12 men and 12 women) were tested over 3 days. Main Outcome Measures: Arterial occlusion pressure (AOP) and the pressure at which the participants rated a 7 out of 10 on the perceived tightness scale in the upper arm and upper leg. Results: The percentage coefficient of variation for the measurement was approximately $12 \%$, with no effect of sex in the upper (median $\delta$ [95\% credible interval]: 0.016 [-0.741, 0.752]) or lower body (median $\delta$ [95\% credible interval]: 0.266 $[-0.396,0.999])$. This would produce an overestimation/underestimation of $\sim 25 \%$ from the mean perceived pressure in the upper body and $\sim 20 \%$ in the lower body. Participants rated pressures above their AOP for the upper body and below for the lower body. At the group level, there were differences in participants' ratings for their relative AOP ( 7 out of 10) between day 1 and days 2 and 3 for the lower body, but no differences between sexes for the upper or lower body. Conclusions: The use of the perceived tightness scale does not provide reliable estimates of relative pressures over multiple visits. This method resulted in a wide range of relative AOPs within the same individual across days. This may preclude the use of this scale to set the pressure for those implementing practical blood flow restriction in the laboratory, gym, or clinic.
\end{abstract}

Keywords: subjectivity, practical, rehabilitation, training

The current literature suggests that the blood flow restriction pressure be set relative to an individual's arterial occlusion pressure (AOP) as this will account for the cuff used and the size of the limb to which the cuff is applied. ${ }^{1}$ However, this method of blood flow restriction is available only to those with the suitable equipment. Others who rely on more practical means of applying pressure (ie, wraps) have often set restriction pressures based on the individual's perception of tightness. ${ }^{2-4}$ Previous research found that subocclusive pressures could be applied when participants rated the level of tightness from the cuff as a 7 out of 10, indicating that this may be a viable method for determination of an appropriate level of cuff restriction. ${ }^{3}$ Additional work on this topic replicated this study with a larger sample size $(n=120)$ and found that the majority of participants rated the 7 out of 10 condition lower than their AOP. ${ }^{5}$ However, both studies only had participants completing one visit, and it was not known whether ratings for a 7 out of 10 pressure are consistent across multiple visits. Therefore, the aim of this study was to discern the reliability of applied pressure when asking participants to rate a 7 out of 10 , considered to be a moderate pressure with no pain over 3 separate visits. We also sought to determine if this differed by sex. ${ }^{6}$

\section{Methods}

All participants were tested at least 2 hours postprandial, and they were also instructed to avoid caffeine 8 hours and alcohol

The authors are with the Department of Health, Exercise Science, and Recreation Management, Kevser Ermin Applied Physiology Laboratory, The University of Mississippi, University, MS. Loenneke (jploenne@olemiss.edu) is corresponding author. and exercise for 24 hours prior to testing for each of their visits. The study received approval from the University of Mississippi's institutional review board, with each participant giving written informed consent before participation. Upon arriving to the laboratory, participants' height and body mass were measured using a standard stadiometer and an electronic scale (Seca, Chino, CA). On average, participants were 23 (3) years, with a height of 171.6 $(8.8) \mathrm{cm}$ and a body mass of $77.2(18.2) \mathrm{kg}$. Separated by sex (12 men and 12 women), men were older (25 [4] vs 22 [1] y), taller (177.2 [6.4] vs 166 [7.2] cm), and had greater body mass (85.9 [13] vs $68.6[19.1] \mathrm{kg})$.

Immediately following measurements for height and body mass, a 5-cm pneumatic cuff was applied to the participants' upper arm and a 10-cm cuff was applied to the participants' upper leg. Cuff allocation was randomized for the side of the body (right or left) along with the order in which region of the body (upper or lower) was measured first. This allocation for side and order was then maintained for visits 2 and 3. Participants were positioned on an examination table with the arm being tested abducted at $90^{\circ}$ on a flat surface, while the nontesting arm relaxed at their side and their legs relaxed and uncrossed for 10 minutes. During the 10-minute rest and the remainder of the session, participants wore headphones that played ambient white noise to decrease the chance that the sounds from the Doppler probe may influence their judgment for the perceived tightness scale. Following 10 minutes of rest in the predetermined randomized order, participants were measured for AOP, and they rated 7 out of 10 on the perceived pressure scale. (Methods for AOP have been outlined previously. ${ }^{5}$ ) When the participants reached a pressure that they deemed to be a moderate pressure with no pain (7 out of 10), they were asked to verbally state the word "7" (in line with the recommendations 
of Wilson et $\mathrm{al}^{3}$ ). In the likelihood that AOP was found before participants rated their 7 out of 10 , inflation of the cuff was continued until the participants did state a 7 out of 10 rating. Conversely, if participants were to rate their 7 out of 10 before AOP was found, the same methods were completed until arterial pressure was found. A 5-minute rest period was given between the measurements of the upper and lower body. Participants were also reminded nonverbally with written signs reiterating how to subjectively assess a 7 (moderate pressure with no pain) out of 10 as the cuff was continually inflated before the 2 measurements. Importantly, the participants could not see the pressure being applied.

The variable of interest was the relative pressure at which participants rated a 7 out of 10 on the perceived tightness scale. A Bayesian repeated-measures analysis with a between-subject factor of sex was used to compare relative AOPs across days and sex with a default prior of 0.5 for the fixed effects. If there was evidence for the alternative hypothesis (Bayes factor $[\mathrm{BF}]_{10}>3$ ), we performed follow-up comparisons across days using a Bayesian paired $t$ test, set to a default Cauchy prior of 0.707 (centered on zero). The percentage coefficient of variation $(\% \mathrm{CV})$ was calculated as the SD between the ratings of relative AOP across time (days 1-3) relative to the mean of the 3 visits to determine the reliability. A Bayesian independent samples $t$ test was used to determine if there were sex differences in \%CV with a default Cauchy prior of 0.707 (centered on zero). Data are presented as mean (SD), unless otherwise stated. Statistical analysis was completed using JASP (version 0.8.6.0; JASP TEAM; Amsterdam, The Netherlands).

\section{Results}

Of the 12 men in the study, 4 were excluded from upper-body analysis because each rated a "7" on the scale at a pressure that

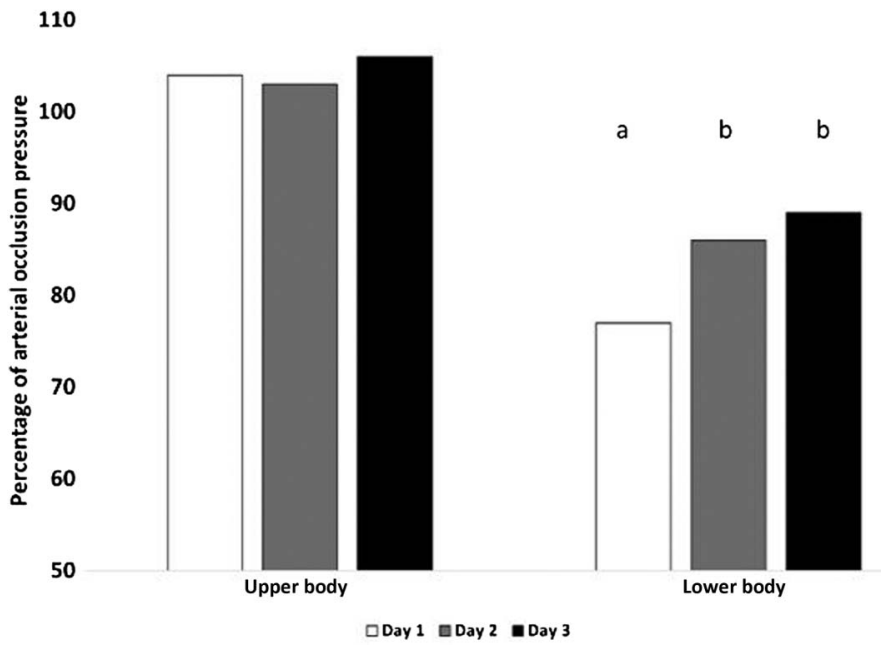

Figure 1 - The relative arterial occlusion pressure across days that corresponded to a 7 out of 10 on the perceived tightness scale. The SD across days was 32, 31, and 38 for the upper body and 27, 26, and 26 for the lower body. Bayes factors $\left(\mathrm{BF}_{10}\right)$ were used to provide evidence for $\left(\mathrm{BF}_{10}\right.$ of $\leq 0.33)$ or against the null $\left(\mathrm{BF}_{10}\right.$ of $\left.\geq 3.0\right)$ hypothesis. As noted in the results, evidence for the null hypothesis was provided in the upper body $\left(\mathrm{BF}_{10}: 0.152\right)$ and evidence for the alternative hypothesis was provided in the lower body $\left(\mathrm{BF}_{10}\right.$ : 17.364). Time points with different letters represent differences in the percentage of arterial occlusion pressure between days. exceeded $300 \mathrm{~mm} \mathrm{Hg}$. For the overall mean values of relative AOP across days (Figure 1), we found evidence toward the null hypothesis with the main effect of day $\left(\mathrm{BF}_{10}: 0.152\right)$ and sex $\left(\mathrm{BF}_{10}: 0.883\right)$ and evidence against the day by sex interaction $\left(\mathrm{BF}_{10}: 0.037\right.$ for interaction model compared with the main effects model of $\mathrm{BF}_{10}:$ 0.114). Individual responses are shown in Figure 2. The $\% \mathrm{CV}$ for relative AOP did not differ between sexes (men: $12.3 \%$ vs women $12.2 \% \mathrm{BF}_{10}: 0.403$; median $\delta$ [95\% credible interval]: $0.016[-0.741,0.752])$. The $\% \mathrm{CV}$ in this study indicates that 2 SD units above or below the mean would result in either an overestimation or an underestimation of $\sim 25 \%$ from the mean perceived pressure.

Of the 12 women in the study, 1 woman was excluded from lower-body analysis as she rated a " 7 " on the scale at a pressure that exceeded $300 \mathrm{~mm} \mathrm{Hg}$. Because of a technical error that occurred on day 3 during the analysis of lower body in 1 woman, \%CV was calculated using data from the other 2 days; however, the woman was excluded from the test of mean ratings across days (12 men and 10 women). For the overall mean values of relative AOP across days (Figure 1), we found evidence for the alternative hypothesis with the main effect of day $\left(\mathrm{BF}_{10}\right.$ : 17.364) but not sex $\left(\mathrm{BF}_{10}\right.$ : $0.629)$. We also found evidence against the day by sex interaction $\left(\mathrm{BF}_{10}: 2.539\right.$ for interaction model compared with the main effects model of $\mathrm{BF}_{10}$ : 11.480). Post hoc analysis suggested that day 1 was different from day $2\left(\mathrm{BF}_{10}: 4.482\right.$, median $\delta[95 \%$ credible interval]: $-0.694[-1.307,-0.130])$ and day $3\left(\mathrm{BF}_{10}: 10.2\right.$, median $\delta$ [95\% credible interval]: $-0.838[-1.468,-0.189])$, but day 2 was not different from day $3\left(\mathrm{BF}_{10}: 0.373\right.$, median $\delta[95 \%$ credible interval]: $-0.264[-0.829,0.260])$. Individual responses are shown in Figure 2. The \%CV for relative AOP did not differ between sexes (men: $13.7 \%$ vs women $10.3 \%$; $\mathrm{BF}_{10}: 0.509$; median $\delta[95 \%$ credible interval]: $0.266[-0.396,0.999])$. The $\% \mathrm{CV}$ in this study indicates that 2 SD units above or below the mean would result in either an overestimation or an underestimation of about $\sim 20 \%$ from the mean perceived pressure.

\section{Discussion}

Findings from our study would suggest blood flow restriction training that relies on practical methods (knee wraps and cuffs) may need to consider alternative approaches for setting the restriction pressure (due to wide range of relative pressures rated to be a "7" and the poor reliability). A future approach with this scale may be to purposively apply a subocclusive pressure, based on a percentage of previously assessed AOP, and state to the participant that this is a target pressure, which should be applied when completing future testing/training. In practice, this approach would require at least one visit with equipment where the pressure is known, with the idea that the participants could be conditioned to associate a number on the perceived tightness scale with the target relative AOP. A notable limitation of this study is that pneumatic cuffs were used instead of practical cuffs or knee wraps, which may slightly alter the perceptual response. However, pneumatic cuffs were necessary as this allowed the researchers to accurately quantify the relative pressures and AOP measurements.

\section{Conclusions}

The perceived tightness scale does not provide reliable estimates of relative pressures over multiple visits. This should be taken into consideration among clinical and recreational gym settings, where blood flow restriction is becoming more widespread. 
A

Men upper (percentage of arterial occlusion pressure)

140

130

120

110

100

90

80

70

60

50

40

C

160

140

120

100

80

60

40

20
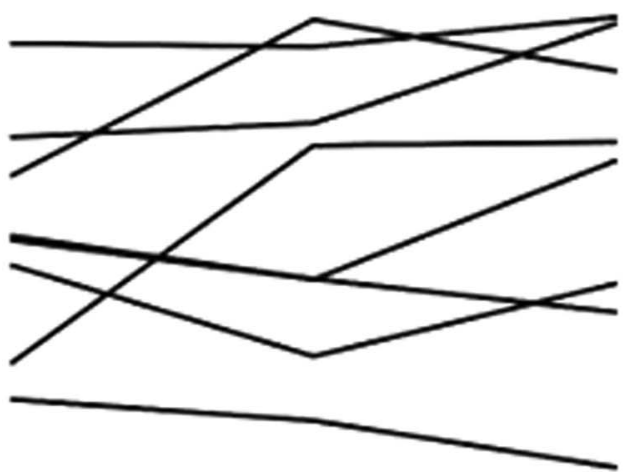

Day 1

Day 2

Day 3
150

100

50

125

115

105

95

85

75

65

55

45

35

25
Women upper (percentage of arterial occlusion pressure)

200

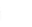

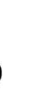

\begin{tabular}{lll}
\hline Day 1 & Day 2 & Day 3
\end{tabular}

Women lower (percentage of arterial occlusion pressure)

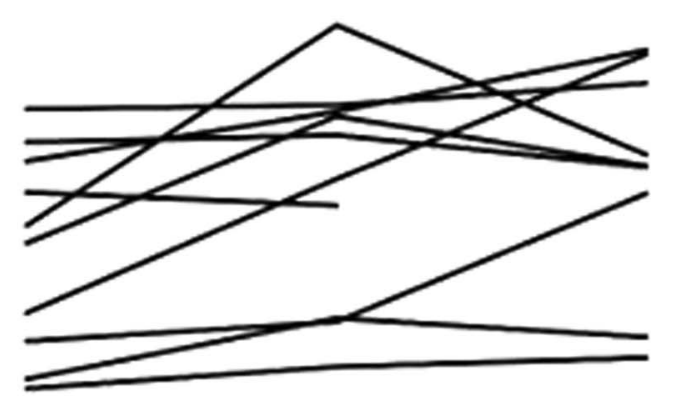

Day 1

Day 2

Day 3

Figure 2 - The relative arterial occlusion pressure across days that corresponded to a 7 out of 10 on the perceived tightness scale separated into individual responses as follows: (A) men upper, (B) women upper, (C) men lower, and (D) women lower. There was a technical error on day 3 of the lower body in one woman; thus, she provided information for days 1 and 2 only.

\section{Acknowledgments}

The authors are not aware of any affiliations, memberships, funding, or financial holdings that might be perceived as affecting the objectivity of this article. The authors have no conflicts of interest to disclose.

\section{References}

1. Mattocks KT, Jessee MB, Counts BR, et al. The effects of upper body exercise across different levels of blood flow restriction on arterial occlusion pressure and perceptual responses. Physiol Behav. 2017; 171:181-186. PubMed ID: 28088558 doi:10.1016/j.physbeh.2017. 01.015

2. Loenneke JP, Kearney ML, Thrower AD, Collins S, Pujol TJ. The acute response of practical occlusion in the knee extensors. $J$ Strength Cond Res. 2010;24(10):2831-2834. PubMed ID: 20885201 doi:10. 1519/JSC.0b013e3181f0ac3a
3. Wilson JM, Lowery RP, Joy JM, Loenneke JP, Naimo MA. Practical blood flow restriction training increases acute determinants of hypertrophy without increasing indices of muscle damage. J Strength Cond Res. 2013;27(11):3068-3075. PubMed ID: 23446173 doi:10.1519/ JSC.0b013e31828a1ffa

4. Lowery RP, Joy JM, Loenneke JP, et al. Practical blood flow restriction training increases muscle hypertrophy during a periodized resistance training programme. Clin Physiol Funct Imaging. 2014; 34(4):317-321. PubMed ID: 24188499 doi:10.1111/cpf.12099

5. Bell ZW, Dankel SJ, Mattocks KT, et al. An investigation into setting the blood flow restriction pressure based on perception of tightness. Physiol Meas. 2018;39(10):105006. PubMed ID: 30211696 doi:10. 1088/1361-6579/aae140

6. Counts BR, Rossow LM, Mattocks KT, et al. Let's talk about sex: where are the young females in blood flow restriction research? Clin Physiol Funct Imaging. 2018;38(1):1-3. PubMed ID: 27730736 doi:10.1111/cpf.12394 